\title{
Indicadores de atraso no desenvolvimento em crianças de creche advindas de famílias de baixa renda
}

\section{Developmental delays among children from day care center of socially disadvantaged families}

\section{I ndicadores de retraso del desarrollo en niños de guarderías provenientes de famílias de baja renta}

\author{
Maria Cristina Triguero Veloz Teixeira* \\ Universidade Presbiteriana Mackenzie, São Paulo, São Paulo, Brasil
}

\author{
Felipe Alckmin-Carvalho** \\ Universidade de São Paulo - USP, São Paulo, São Paulo, Brasil
}

Deisy Ribas Emerich***

Universidade de São Paulo - USP, São Paulo, São Paulo, Brasil

Priscilla Veloz Cevallos****

Universidade Presbiteriana Mackenzie, São Paulo, São Paulo, Brasil

\section{Cristiane Silvestre de Paula*****}

Universidade Presbiteriana Mackenzie, São Paulo, São Paulo, Brasil

\begin{abstract}
RESUMO
Avaliações periódicas precoces de indicadores de desenvolvimento são importantes para a elaboração de programas preventivos. O objetivo deste estudo foi identificar atrasos em indicadores globais de desenvolvimento em 100 crianças (idades entre 16-24 meses, média de 21,84 meses, $\mathrm{DP} \pm 1,94$ ), matriculadas em creches de um município do Estado de São Paulo. 0 instrumento de coleta de dados foi o Teste de Triagem de Desenvolvimento de Denver II (Denver II). Na amostra estudada, o domínio de maior prejuízo foi o da linguagem, seguido pelo domínio de cuidado pessoal-social. Os resultados do estudo apontam para a necessidade de incluir na avaliação do desenvolvimento infantil fatores familiares e educacionais comumente associados a atrasos de desenvolvimento.
\end{abstract}

Palavras-chave: creches, desenvolvimento, Denver II, Estudo Transversal.

\section{ABSTRACT}

Periodic early assessments of child development are important to elaborate prevention programs. The goal of this study was to identify global developmental delays in 100 children (aged 16-24 months, mean age 21.84 months, $\mathrm{SD} \pm 1.94$ ) who attended day care centers in a town in the São Paulo 
State. The used instrument was the Denver Developmental Screening Test II (Denver II). In the current sample, greatest deficits were observed in the speech/language domain, followed by the social-emotional development domain. The study results indicate the need of including familial/educational factors into child development's assessments, which are commonly associated with developmental delays.

Keywords: day care centers, development, Denver II, Cross Sectional Study.

\begin{abstract}
RESUMEN
Evaluaciones periódicas tempranas de indicadores de desarrollo son importantes para la creación de programas de prevención. El objetivo de este estudio fue identificar retrasos en indicadores globales de desarrollo en 100 niños (de 16 a 24 meses, promedio 21,84 meses, SD $\pm 1,94$ ), asistidos en guarderías en una ciudad de São Paulo. El instrumento de recolección de datos fue el teste de Evaluación del desarrollo Denver II (Denver II). En esta muestra, la mayor pérdida fue el dominio de la lengua, seguida del dominio de la atención personal-social. Los resultados del estudio apuntan para la necesidad de incluir en la evaluación del desarrollo infantil factores ambientales familiares y educacionales comunmente asociados a atrasos del desarrollo.
\end{abstract}

Palabras-clave: guarderías, desarrollo, Denver II, Estudio Transversal.

\title{
I ntrodução
}

O desenvolvimento infantil é o resultado de uma complexa interação entre fatores pré-natais ambientais e hereditários e fatores pósnatais biológicos, culturais, sociais e educacionais (Gallahue, Ozmun, \& Goodway, 2013; Miller, Maguire, \& Macdonald, 2011; Peacock, Konrad, Watson, Nickel, \& Muhajarine, 2013; Veleda, Soares, \& Cézar-Vaz, 2011). Está bem estabelecida na literatura sobre desenvolvimento infantil a necessidade de realizar avaliações periódicas de indicadores de desenvolvimento na criança, desde o nascimento, tendo, a maior parte delas, fins preventivos ou para o diagnóstico precoce de transtornos que afetam o desenvolvimento (Carvalho, Paula, Teixeira, Zaqueu, \& D'antino, 2013; Räikkönen, Pesonen, Roseboom, \& Eriksson, 2012; Souza, Gouveia, Almeida, \& Torres, 2014). Nessas avaliações recomenda-se o uso de testes que tenham indicadores padronizados sobre habilidades motoras, habilidades cognitivas e de linguagem, indicadores de funcionamento em atividades de vida diária, comportamento e comunicação (Shevell, 2010), uma vez que tais áreas são importantes marcos do desenvolvimento e caracterizam o desenvolvimento neuropsicomotor psicossocial de crianças nos primeiros cinco anos de vida.

Estudos de rastreamento reportam a identificação de algum tipo de atraso e/ou alteração no desenvolvimento desde idades precoces, confirmando a necessidade de avaliações periódicas do neurodesenvolvimento infantil com fins preventivos (Chiu \& DiMarco, 
2010; Sajedi, Vameghi, \& Kraskian-Mujembari, 2014; Valla, WentzelLarsen, \& Hofoss 2015). Um dos locais que podem servir como lócus para a realização dessas avaliações são as creches, uma vez que essas unidades permitem rastrear indicadores de desenvolvimento infantil em números populacionais expressivos, o que facilita a identificação precoce de casos vulneráveis e, por consequência, a implementação de medidas em nível de prevenção, promoção e tratamento (Barros, Carvalho, Franco, Mendonça, \& Rosalém, 2011; Ramos \& Salomão, 2013).

No primeiro ano de vida, o desenvolvimento infantil sofre maior influência de fatores maturacionais e/ou biológicos. Entretanto, a partir do segundo ano a influência de fatores ambientais de tipo social, cultural e educacional aumenta expressivamente (Magalhães, Fonseca, Martins, \& Dornellas, 2011). É consenso na literatura que as características do ambiente onde a criança está inserida, bem como a qualidade de sua interação com ambiente e com outras crianças e adultos terão impacto direto nos indicadores de desenvolvimento (Chang, Lichtenstein, Asherson, \& Larsson, 2013; Räikkönen, Pesonen, Roseboom, \& Eriksson, 2012; Scher, 2013; Shan, Muhajarine, Loptson, \& Jeffery, 2012).

O impacto de fatores ambientais sobre o desenvolvimento infantil tem sido estudado em crianças com desenvolvimento típico e atípico. Por exemplo, estudos apontam que crianças que já apresentam algum nível de comprometimento e que vivem em ambientes ricos em estimulações cognitivas e sociocomportamentais adequadas costumam ter melhores prognósticos quando comparadas àquelas que crescem em condições adversas (Dawson, 2013). Tal dado é reforçado por estudos longitudinais que têm evidenciado a efetividade das intervenções multimodais em saúde mental com a finalidade de reduzir fatores de risco, promover fatores de proteção ao desenvolvimento infantil, de modo a minimizar posteriores comprometimentos na qualidade de vida do indivíduo e de sua família (Belini \& Fernandes, 2007; Chiu \& DiMarco, 2010). Neste sentido, a identificação precoce de atrasos de desenvolvimento na primeira infância, associada ao tratamento intensivo são de grande relevância e essas medidas estão associadas a impacto positivo no prognóstico (Carvalho et al., 2013; Valla et al., 2015).

Um dos instrumentos internacionalmente reconhecidos para quantificar e monitorar possíveis atrasos de desenvolvimento nas áreas de linguagem, pessoal-social e habilidades motoras em crianças desde o nascimento até os seis anos de idade é o Teste de Triagem de Desenvolvimento de Denver II (Development Screening Test-II DENVER II) (Frankenburg et al., 1990). O teste já foi traduzido e adaptado para a língua Portuguesa (Figueiras, Pedromônico, Sales, \& Figueiras, 2000), porém ainda não foram divulgados dados com 
evidências de validade do instrumento para a população brasileira (Custódio, Crepaldi, \& Cruz, 2012; Magalhães et al., 2011).

Estudos anteriores realizados no Brasil têm utilizado o DENVER II para avaliação de crianças que apresentam sinais precoces de Transtornos do Espectro do Autismo, crianças nascidas pré-termo e com baixo peso, crianças expostas a intervenções nutricionais e cirúrgicas, crianças com doenças crônicas e crianças expostas a fatores de risco pré-natal e ambientais associados à baixa renda familiar (Custódio et al., 2012; Fisberg et al., 1997; Magalhães et al., 2011; Zaqueu, Teixeira, Alckmin-Carvalho, \& Paula, 2015).

Reconhecendo o impacto positivo que as avaliações preventivas podem ter sobre a qualidade de vida de crianças com atraso no desenvolvimento neuropsicomotor e de sua família, faz-se necessário investir em estudos de rastreamento de indicadores no desenvolvimento neuropsicomotor na infância. O estudo teve como objetivo identificar atrasos de indicadores globais do desenvolvimento em crianças de creches advindas de famílias de baixa renda.

\section{Método}

\section{Participantes}

Foram incluídas no estudo 100 crianças com idade entre 16 e 24 meses (Média $=21,84, \mathrm{DP}= \pm 1,94)$, sendo a maioria meninos $(53,0 \%)$ e suas respectivas assistentes maternais. O critério de inclusão destas foi exercer o cuidado das crianças por, pelo menos três meses, antes da avaliação e no mínimo exercer o cuidado por seis horas diárias. Todas as crianças estavam regularmente matriculadas e frequentavam creches públicas de Barueri, município localizado na região metropolitana de São Paulo. Na ocasião da coleta de dados a rede da cidade contava com 22 creches que forneciam atendimento a 4.502 crianças. A amostra deste estudo foi coletada em cinco dessas unidades. A maioria dos participantes apresentava renda familiar entre um e quatro salários mínimos, com média de 1,55 salários mínimos, dado que classifica essas famílias no estrato socioeconômico baixo de acordo com o DIEESE (Departamento Intersindical de Estatística e Estudos Socioeconômicos).

Os critérios de exclusão foram: crianças diagnosticadas com malformações, doenças neurológicas, síndromes genéticas, doenças agudas, queixas de fala, audição e linguagem (tanto expressiva como receptiva). Essas variáveis foram verificadas no prontuário de cada criança na creche. 


\section{Instrumento}

O instrumento utilizado neste estudo foi o Teste de Triagem de Desenvolvimento de Denver II (Development Screening Test-II DENVER II) (Frankenburg et al., 1990). Foi elaborado por profissionais da saúde e pode ser aplicado a crianças de 0 a 6 anos. 0 instrumento é indicado para rastreamento de indicadores globais de desenvolvimento infantil e de atraso e/ou suspeita de atraso, composto por 125 itens. Sua aplicação é individual e relativamente rápida (aproximadamente 20 minutos). Optou-se pelo teste Denver por ser um instrumento desenvolvido para fins de rastreamento de indicadores de desenvolvimento em populações infantis sem queixas de atrasos.

O Denver II avalia o desenvolvimento infantil a partir do relato de cuidadores e da observação direta do comportamento da criança durante a realização de tarefas estruturadas. Os comportamentos e habilidades são avaliados em quatro áreas: (1) pessoal-social; (2) motor fino adaptativo; (3) linguagem e (4) motor grosso (Frankenburg et al., 1990). No estudo foram utilizados 37 dos 125 itens do teste (todos aqueles destinados à avaliação de crianças com idade na faixa de 16 a 24 meses), sendo 14 itens direcionados aos cuidadores e 23 realizados com a própria criança. Os itens direcionados aos cuidadores foram respondidos pelas assistentes maternais.

A correção do teste foi feita seguindo o manual do Denver II. A saber, na folha de reposta foi registrada a porcentagem do quanto a criança era capaz de realizar as atividades propostas (25, 50, 75 e 90\%) com base na amostra normativa de crianças americanas. A interpretação individual dos itens ocorreu da seguinte forma: (a) Item avançado quando a criança passa em um item que se encontra, completamente, à direita da linha de idade da criança; (b) Item normal - quando a criança falha ou recusa em um item que está completamente à direita da linha de idade e quando a criança passa, falha ou recusa em um item que se encontra na linha de idade entre o percentil 25 e 75; (c) Item precaução - quando a criança falha ou recusa em um item que se encontra na linha de idade entre 0 percentil 75 e 90; (d) Item atraso - quando a criança falha ou recusa em um item que está completamente à esquerda da linha de idade; (e) Item não observado - quando as assistentes maternais comunicaram que não houve oportunidade para avaliar este item na criança. Quando verificado esse último tipo de item, o mesmo não foi considerado na interpretação global do teste.

Para a classificação do desenvolvimento foi adotada a normatização do manual do teste (Frankenburg et al., 1990) que sugere uma interpretação final em termos de: (a) Normal - crianças que apresentam no máximo um item em precaução e nenhum na 
categoria atraso; (b) Suspeita de atraso - crianças que apresentam dois ou mais itens pontuados como precaução e um ou mais itens pontuados como atraso; (c) Não testável - crianças que recusam em um ou mais itens que estão completamente à esquerda da linha de idade ou, acima de um item que cruza a linha de idade numa área de $75 \%-90 \%$.

\section{Procedimentos de coleta e aspectos éticos}

A realização desta pesquisa foi aprovada pelo Comitê de Ética em Pesquisa com Seres Humanos da Universidade Presbiteriana Mackenzie, protocolo CAAE número 0005.0.272.000-09. Os dados do estudo foram coletados durante o horário de funcionamento das creches, a fim de minimizar o impacto da pesquisa na rotina de trabalho das instituições. A observação direta do comportamento das crianças foi realizada em sala privativa, com controle rigoroso de estímulos visuais e auditivos que pudessem interferir no desempenho no teste, composta por mesa e cadeira para a pesquisadora e mesa pedagógica com cadeira adequada à criança. Todas as crianças foram avaliadas individualmente e o tempo de duração foi de aproximadamente 30 minutos com cada uma delas.

Durante a avaliação direta, as crianças eram acompanhadas pelas assistentes, que permaneciam afastadas e em silêncio durante a avaliação. Para as crianças que manifestaram algum indicador comportamental de recusa, a coleta dos dados foi interrompida, de modo a respeitar o seu desejo de não continuar a avaliação. Posteriormente, em sala privativa, durante aproximadamente 15 minutos, as assistentes maternais responderam individualmente aos itens previstos no teste em relação à criança. As crianças foram avaliadas por dois examinadores treinados seguindo as orientações do manual de aplicações do Denver II (Frankenburg et al., 1990).

As equipes educacionais das creches e os pais das crianças participantes do estudo receberam relatórios de devolutiva em que estavam identificadas as áreas mais prejudicadas do desenvolvimento infantil, assim como os itens em que as crianças mais frequentemente apresentaram problemas. Especialistas em Distúrbios do Desenvolvimento vinculados à instituição onde foi feita a pesquisa realizaram, em uma das creches, uma palestra com recomendações sobre como prover estimulação precoce em cada um dos domínios avaliados, minimizando o impacto de possíveis atrasos no desenvolvimento.

Por fim, as crianças que tiveram suspeitas de atraso no desenvolvimento foram acompanhadas pela equipe de especialistas, a fim de verificar o desfecho de cada caso. Os dados dessa segunda etapa do estudo podem ser consultados em Alckmin-Carvalho, Teixeira, Brunoni, Strauss e Paula (2014). 
Análise de dados

A análise dos dados foi realizada no programa SPSS, versão 17.0. Foram conduzidas análises de frequência para verificar o número de habilidades em atraso de acordo com as escalas do teste e identificar os casos com atrasos globais de desenvolvimento. O teste Z-score foi utilizado para avaliar diferenças de proporções entre grupos (normal e com suspeita de atraso no desenvolvimento) em cada um dos itens avaliados. Foi utilizado o teste $t$ de student para comparação das médias de idade entre os grupos, bem como o teste Qui-quadrado $\left(x^{2}\right)$ para verificar possíveis associações entre suspeita de atraso no desenvolvimento e renda familiar, expressa nos seguintes níveis categóricos de salários mínimos: um a dois salários; três a quatro; cinco a dez e mais de dez salários.

\section{Resultados}

$\mathrm{Na}$ avaliação foi verificado um percentual elevado de crianças com suspeita de atrasos no desenvolvimento $(n=30 ; 30,00 \%)$. As crianças do grupo com suspeita tinham idade variando entre 17 e 24 meses (Média $=22,57$; Desvio Padrão $= \pm 1,76$ ). Para grupo em que não se observou indicadores de atraso (Grupo Normal), a faixa etária das crianças foi bastante similar, entre 16 e 24 meses de idade (Média= 21,53; Desvio padrão $= \pm 1,94)$. O Teste $t$ de Student para comparação de médias de idade entre o grupo com suspeita de atraso e o grupo normal não verificou diferenças estatisticamente significativas. Os dados sobre a distribuição das falhas avaliadas, de acordo com o grupo, segundo as áreas de desenvolvimento e cada item do teste estão descritos na Tabela 1. 
Maria Cristina Triguero Veloz Teixeira, Felipe Alckmin-Carvalho, Deisy Ribas Emerich, Priscilla Veloz Cevallos, Cristiane Silvestre de Paula

Tabela 1

Distribuição dos grupos de acordo com as falhas por áreas e itens do Denver II

\begin{tabular}{|c|c|c|c|c|c|c|}
\hline \multirow[b]{2}{*}{ Ärea } & \multirow[b]{2}{*}{ Itens Avaliados } & \multicolumn{2}{|c|}{$\begin{array}{l}\text { Grupo normal } \\
\qquad(n=70)\end{array}$} & \multicolumn{2}{|c|}{$\begin{array}{c}\text { Grupo com } \\
\text { suspeita }(n=30)\end{array}$} & \multirow[t]{2}{*}{ Z-score } \\
\hline & & $\mathrm{N}$ & $\%$ & $\mathrm{~N}$ & $\%$ & \\
\hline \multirow{9}{*}{$\begin{array}{l}\text { Pessoal- } \\
\text { Social }\end{array}$} & 1. Se a criança bebe do copo & 00 & 00 & 00 & 00 & - \\
\hline & 2. Se a criança imita atividades & 00 & 00 & 01 & 3,30 & -1.5352 \\
\hline & 3. Se a criança ajuda em casa & 01 & 1,40 & 01 & 3,30 & -0.6235 \\
\hline & 4. Se a criança usa garfo e colther & 00 & 00 & 01 & 3,30 & -1.5352 \\
\hline & 5. Se a criança tira o vestuário & 05 & 7,10 & 07 & 23,30 & $-2.2832^{*}$ \\
\hline & 6. Se a criança alimenta a boneca & 03 & 4,40 & 02 & 6,70 & -0.5006 \\
\hline & 7. Se a criança a escova os dentes com & 10 & 16,90 & 04 & 13,80 & 0.1258 \\
\hline & 8. Se a criança lava e seca as mãos & 24 & 34,30 & 11 & 36,70 & -0.2288 \\
\hline & 9. Se a criança veste a roupa sozinha & 34 & 48,60 & 19 & 63,30 & -1.3554 \\
\hline \multirow{6}{*}{$\begin{array}{l}\text { Motor Fino- } \\
\text { Adaptativo }\end{array}$} & $\begin{array}{l}\text { 1. Se a criança coloca os blocos dentro da } \\
\text { caneca }\end{array}$ & 00 & 00 & 00 & 00 & - \\
\hline & 2. Se a criança rabisca & 00 & 00 & 02 & 6,70 & -2.1822 \\
\hline & 3. Joga fora sementes, como demonstrado & 00 & 00 & 02 & 6,90 & $-2.1822^{*}$ \\
\hline & 4. Montar torre de 2 cubos & 00 & 00 & 02 & 6,90 & $-2.1822^{*}$ \\
\hline & 5. Montar torre de 4 cubos & 09 & 12,90 & 10 & 33,30 & $-2.3919^{*}$ \\
\hline & 6. Montar torre de 6 cubos & 35 & 50,00 & 19 & 63,30 & -1.2259 \\
\hline \multirow{13}{*}{ Linguagem } & 1. Se a criança diz 1 palavra & 00 & 00 & 01 & 3,30 & -1.5352 \\
\hline & 2. Se a criança diz 2 palavras & 00 & 00 & 01 & 3,30 & -1.5352 \\
\hline & 3. Se a criança diz 3 palavras & 00 & 00 & 07 & 23,30 & $-4.1908^{x}$ \\
\hline & 4. Se a criança diz 6 palavras & 02 & 2,90 & 17 & 56,70 & $-6.2856^{*}$ \\
\hline & 5. Se a criança combina palavras & 13 & 18,60 & 18 & 60,00 & $-4.1049^{*}$ \\
\hline & 6. Fala parcialmente compreensivel & 09 & 12,90 & 11 & 36,70 & $-2.7277^{x}$ \\
\hline & 7. Se a criança aponta 2 figuras & 03 & 4,30 & 07 & 23,30 & $-2.9096^{x}$ \\
\hline & 8. Se a criança nomeia 1 figura & 11 & 15,90 & 07 & 23,30 & -0.9088 \\
\hline & 9. Nomeia 6 partes do corpo & 12 & 17,10 & 07 & 24,10 & -0.7231 \\
\hline & 10. Se a criança aponta 4 figuras & 22 & 31,40 & 15 & 50,00 & -1.7627 \\
\hline & 11. Se a criança nomeia 4 figuras & 37 & 52,90 & 22 & 78,60 & -1.9078 \\
\hline & 12. Se a criança sabe falar 2 ações & 13 & 19,70 & 18 & 64,30 & $-4.1049^{*}$ \\
\hline & 13. Fala de modo compreensivel & 31 & 47,70 & 22 & 78,60 & $-2.6671^{*}$ \\
\hline \multirow{9}{*}{$\begin{array}{l}\text { Motor } \\
\text { Grosso }\end{array}$} & 1. Se a criança fica em pé sozinha & 00 & 00 & 01 & 3,30 & -1.5352 \\
\hline & 2. Se a criança inclina e depois levanta & 00 & 00 & 01 & 3,30 & -1.5352 \\
\hline & 3. Se a criança caminha bem & 00 & 00 & 01 & 3,30 & -1.5352 \\
\hline & 4. Se a criança anda para atrás & 00 & 00 & 02 & 6,70 & $-2.1822^{x}$ \\
\hline & 5. Se a criança corre & 00 & 00 & 01 & 3,30 & -1.5352 \\
\hline & 6. Se a criança sobre degraus & 00 & 00 & 01 & 3,30 & -1.5352 \\
\hline & 7. Se a criança chuta bola para frente & 01 & 1,40 & 03 & 10,00 & $-2.0045^{*}$ \\
\hline & 8. Arremessa a bola sobre os ombros & 08 & 11,40 & 03 & 10,30 & 0.2092 \\
\hline & 9. Se a criança salta & 00 & 00 & 04 & 13,30 & $-3.118^{*}$ \\
\hline
\end{tabular}

O domínio Motor Grosso do teste foi o menos afetado entre os participantes avaliados, havendo diferenças estatisticamente significativas entre o grupo normal e com suspeita de atraso em apenas três provas, a saber: se a criança anda para atrás, chuta bola para frente e salta. De modo geral, a maioria das crianças, de ambos os grupos, conseguiram cumprir as tarefas relativas a esse domínio. Apenas nove $(12,85 \%)$ crianças do grupo normal falharam nos itens relacionados ao domínio Motor Grosso, enquanto, no grupo com suspeita de atraso no desenvolvimento, foram $17(56,66 \%)$, sendo que o item com maior comprometimento foi o de arremessar a bola sobre os ombros. 
No domínio Pessoal-Social mais de $30 \%$ da amostra, independente do grupo, falhou nos itens lavar e secar as próprias mãos e vestir-se sozinha, sendo esse último item o mais comprometido no grupo com suspeita de atraso no desenvolvimento. Em nenhum dos dois itens houve diferença estatisticamente significativa entre os grupos. 0 único item em que foram identificadas diferenças estatisticamente significativas entre proporções nos grupos no domínio Pessoal-Social foi o referente a tirar o vestuário, em que cinco $(7,10 \%)$ crianças do grupo normal falharam e sete $(23,3 \%)$ do grupo com suspeita de atraso.

Em relação às habilidades motoras finas adaptativas, as tarefas montar uma torre com quatro e seis cubos foram as que evidenciaram os maiores comprometimentos em ambos os grupos. A maior proporção de crianças com falhas na tarefa de montar uma torre com quatro e seis cubos foi observada no grupo com suspeita de atraso $(63,30 \%)$. Contudo, no grupo normal o percentual de falhas também foi elevado, pois metade deles tampouco completou a tarefa.

O domínio no qual observaram-se maiores déficits, considerando a amostra total, foi o de linguagem. Em percentual, as crianças do grupo com suspeitas de atraso no desenvolvimento falharam mais em todas as provas, em comparação ao grupo normal, havendo diferenças estatisticamente significativas nas seguintes provas: se a criança diz três palavras, combina seis palavras, tem fala parcialmente compreensível, aponta duas figuras, sabe falar duas ações, e se a criança fala de modo compreensível. As únicas tarefas nas quais o grupo normal não mostrou falhas foram falar uma a três palavras. Já nos itens restantes nesse grupo se verificou que pelo menos três crianças falharam em todas as tarefas, sendo as mais prejudicadas apontar quatro figuras, nomear quatro figuras e falar de modo compreensível.

Por fim, foi utilizado o teste Qui-quadrado para verificar possíveis associações entre a variável relativa ao desenvolvimento (com e sem suspeita de atraso) e a renda familiar, essa última expressa nos seguintes níveis de salários mínimos (um a dois salários; três a quatro; cinco a dez e mais de dez salários). A análise dos dados apontou que não houve diferenças estatisticamente significativa entre os grupos $\left(x^{2}=0,27, p=0,96\right)$.

\section{Discussão}

Os resultados do estudo revelaram que $30 \%$ dos participantes avaliados apresentam indicadores de desenvolvimento aquém do esperado para a idade. Esse percentual de crianças com suspeita de atraso no desenvolvimento é semelhante ao reportado em outros dois 
estudos brasileiros que também utilizaram o teste Denver (Halpern, Giugliani, Victora, Barros, \& Horta, 2000; Biscegli, Polis, Santos, \& Vicentin, 2007). O estudo de Halpern e colaboradores foi desenvolvido em Pelotas, Rio Grande do Sul, em uma amostra de 1.362 crianças de 12 meses de idade, selecionada aleatoriamente de uma coorte populacional formada por 5.304 crianças que nasceram nos hospitais de Pelotas e que residiam na zona urbana. No estudo foi verificado que $34 \%$ das crianças tinham atraso de desenvolvimento (Halpern et al., 2000). Em Catanduva, Estado de São Paulo, Biscegli et al. (2007) identificaram uma taxa de 37\% de atraso em 113 crianças com idades entre seis e 70 meses de idade.

Estudos realizados em cidades nordestinas identificaram taxas de atraso de desenvolvimento ainda superiores às verificadas em nosso estudo. Em uma amostra de 398 crianças acompanhadas em Unidade Básicas de Saúde da cidade de Natal, com idades entre 0 e 12 meses, Cunha (2008) identificou suspeita de atraso em 182 casos $(45,72 \%)$. Um percentual maior de atrasos de desenvolvimento foi detectado em estudo seccional com censo da população de crianças na faixa etária entre seis e 18 meses provenientes de berçários de Centros de Referência em Educação Infantil da Rede Municipal de Ensino da cidade de João Pessoa. Nesse estudo identificou-se que 59 crianças $(52,7 \%)$ de uma amostra de 112 apresentavam atraso no desenvolvimento (Silva, Engstron, \& Miranda, 2015).

Somente dois dos estudos citados anteriormente utilizaram amostras provenientes de coortes populacionais (Halpern et al., 2000; Silva et al., 2015). Os outros dois estudos adotaram amostragens não probabilísticas (Biscegli et al., 2007; Cunha, 2008). Contudo, independentemente dos critérios de seleção amostral, as taxas de suspeita de atraso de desenvolvimento detectadas nos grupos estudados são maiores nas crianças provenientes da região nordestina, sobretudo no estudo de Silva e colaboradores, cujos participantes foram crianças de creches. Alguns dos achados desses estudos revelaram uma associação entre essas taxas de atrasos e variáveis ambientais como nível de escolaridade materna e paterna, renda familiar, fatores de risco pré e pós-natais, acompanhamento por puericultura, dentre outros.

Tanto quanto permite 0 alcance desta pesquisa, podem ser levantadas hipóteses de fatores ambientais e socioeconômicos familiares, bem como de fatores relacionados ao contexto das próprias creches para entender o índice de suspeitas de atraso no desenvolvimento que foi verificado. Para entender o desenvolvimento infantil é essencial que sejam contempladas nessa compreensão, fatores culturais, econômicos e sociais, condições familiares, qualidade da assistência recebida pelos serviços de educação e saúde, dentre outros (Guimarães, Carvalho, Machado, Baptista, \& Lemos, 2013; Silva, et al., 2015; Valla et al., 2015). 
Em relação aos fatores contextuais das próprias creches caberia destacar que a amostra de crianças participantes de nosso estudo convive aproximadamente oito horas por dia nessas instituições. Espera-se que as mesmas recebam, nesse contexto, as estimulações previstas nos currículos educacionais nas áreas cognitiva, motricidade, linguagem, comportamento, habilidades básicas da vida diária, etc., tal como preconizado em parâmetros nacionais da Educação Infantil (Brasil, 2010). Com relação a esses indicadores, um trabalho anterior avaliou 21 das 22 creches do município, das quais cinco creches fizeram parte de nosso estudo (Figueirêdo, 2010). No estudo, as creches foram avaliadas de acordo com ambiente físico, saúde, segurança, nutrição, refeições, administração, interação técnico-família, interação técnico-criança, comportamento observável das crianças e currículo. Para isso foi utilizado o instrumento da Organização Mundial da Saúde 'Child Care Facility Schedule' (Figueirêdo, 2010). Os resultados revelaram que, apesar das creches estarem dentro dos padrões esperados de qualidade, estabelecidos no instrumento, em algumas áreas havia dificuldades como por exemplo, números excessivos de crianças nas salas, bem como número insuficiente de assistentes maternais em relação ao número de crianças. Discutiu-se no estudo se essa proporção garantiria uma estimulação adequada das crianças e a implementação dos currículos educacionais com a qualidade esperada (Figueirêdo, 2010).

Sabe-se, na Psicologia do Desenvolvimento, que a identificação precoce de atrasos no desenvolvimento ou fatores de risco para transtornos do neurodesenvolvimento devem ser prioridade nas políticas públicas de educação e saúde (Couto, Duarte, \& Delgado, 2008). Entretanto, um fator ambiental relativo à família identificado neste estudo também deve ser objeto de discussão. Mais da metade das crianças tinha renda familiar entre um e dois salários mínimos (equivalente, no momento do estudo, a $R \$ 465,00$ até $R \$ 930,00$ ). Cabe levantar a hipótese que, associado a essa baixa renda, deva prevalecer baixa escolaridade parental, que provavelmente influencia negativamente na qualidade da estimulação que essa criança recebe no ambiente familiar.

No presente estudo, o domínio mais prejudicado foi o da linguagem, tanto no grupo normal como entre as crianças com suspeitas de atraso no desenvolvimento. Resultados semelhantes, com hipóteses explicativas semelhantes, também, foram divulgados em estudos anteriores (Ribeiro, Perosa, \& Padovani, 2014; Sabatés \& Mendes, 2007; Silva et al., 2015). Por exemplo, o comprometimento da área de linguagem foi identificado em $84 \%$ das crianças (idade entre 12 e 36 meses) com suspeita de atraso no desenvolvimento em estudo realizado em Guarulhos-SP (Sabatés \& Mendes, 2007) e em 24,6\% das crianças (idade entre 11 e 12 meses) com suspeita de atraso avaliadas em um município de médio porte, com 120.000 habitantes, 
localizado na região centro-oeste do interior do estado de São Paulo (Ribeiro et al., 2014). Do mesmo modo, a linguagem foi a área com maior comprometimento para a amostra de João Pessoa, havendo atrasos para $22(19,6 \%)$ das 112 crianças avaliadas (Silva et al., 2015).

Apesar das discrepâncias encontradas em estudos brasileiros referentes a taxas de déficits e atrasos de desenvolvimento (Biscegli et al., 2007; Chiu \& DiMarco, 2010; Cunha, 2008; Halpern et al., 2000; Rezende, Beteli, \& Santos, 2005; Rezende, Costa, \& Pontes, 2005; Ribeiro et al., 2014; Sabatés \& Mendes, 2007; Silva et al., 2015; Souza \& Siqueira, 2003) há um aspecto que é comum entre esses estudos e trabalhos de outros países com crianças de classes socioeconômicas desfavorecidas (Aly, Taj, \& Ibrahim, 2010; Sajedi, et al., 2014): a área da linguagem tende a ser a mais comprometida.

As 30 crianças com suspeita de atraso no desenvolvimento identificadas neste estudo tiveram idades entre 17-24 meses. $E$, dessas 30 crianças, 18 não foram capazes de dizer ao menos seis palavras e 23 falharam ao nomear quatro figuras. Estes dados revelam atrasos na capacidade expressiva das crianças, pois próximo aos 18 meses o esperado é que a criança tenha um repertório de pelo menos dez palavras por mês e, entre os 17 e 20 meses, um repertório estimado de 50 palavras (Hawa \& Spanoudis, 2014). Sabese que existe uma correlação entre a área da linguagem e o uso de comunicação gestual em crianças (Bates \& Dick, 2002), por isso muitas vezes as crianças com atraso no desenvolvimento da linguagem utilizam gestos para compensar os déficits nas habilidades de linguagem expressiva (Thal \& Tobias, 1994). Na presente amostra também observamos este padrão, uma vez que 50\% das crianças com suspeita de atraso na área da linguagem também falharam na tarefa de apontar (quatro) figuras.

É necessário entender como as habilidades de linguagem, assim como outras áreas do desenvolvimento dependem das oportunidades oferecidas pelo ambiente para seu desenvolvimento pleno (Mendes, Pandolfi, Carabetta Júnior, Novo, \& Colombo-Souza, 2012). Estudo recém-publicado, de Costa, Cavalcante e Dell'Aglio (2015), que também utilizou o teste Denver II para verificação de atrasos do desenvolvimento, revelou que os atrasos maiores foram na área de linguagem associados a fatores familiares como baixa escolaridade e nível socioeconômico, além de fatores de estimulação no contexto das unidades de educação infantil. Nesse trabalho os autores levantam os seguintes fatores possivelmente associados aos prejuízos na área de linguagem: nível de pobreza associado significantemente com a suspeita de atraso na linguagem, baixo grau de escolaridade e nível socioeconômico de profissionais da educação infantil e uso de estilos linguísticos mais simples e empobrecidos, proporção 
educadora-criança, déficits na capacitação e formação permanente das profissionais e a responsividade interpessoal (Costa et al., 2015). A preocupação com o desenvolvimento de diversas habilidades, dentre elas a de linguagem, está explicita nas Diretrizes Curriculares Nacionais para a Educação Infantil, estabelecidas pelo Ministério da Educação (MEC) (Brasil, 2010). De um lado, práticas pedagógicas em creches e pré-escolas devem ter como eixos norteadores as interações e a brincadeira com vistas a garantir às crianças, entre outros aspectos, "[...] experiências de narrativas, de apreciação e interação com a linguagem oral e escrita, e convívio com diferentes suportes e gêneros textuais orais e escritos" (Brasil, 2010, p. 25). De outro, devem ser considerados também outros fatores que vão além dos currículos educacionais, como discutido na área educacional contemporânea. Nesse sentido Fonseca (2014) enfatiza que para enriquecer diversas funções e áreas de desenvolvimento, a interação do professor/educador-aluno tem que ser mais intensa e intencional, focando desafios cognitivos, conativos e executivos. E, ainda, o autor especifica que não está em jogo o enriquecimento curricular, está mais em jogo o enriquecimento do potencial de aprendizagem do aluno (Fonseca, 2014).

A aquisição de habilidades de linguagem é um processo complexo determinado por uma associação entre variáveis biológicas e ambientais, que se influenciam de modo bidirecional, ou seja, ambientes ricos em estimulação e que propiciam às crianças acesso a diferentes modalidades de atividades favorecem o desenvolvimento biológico/cognitivo, que por sua vez, possibilita a aquisição dessas habilidades. Uma vez que o desenvolvimento infantil é influenciado por determinantes de diferentes ambientes nos que a criança está inserida desde o nascimento, dificuldades na implementação de estimulações para a aquisição de linguagem poderiam ser avaliadas como fatores de risco (e não causa) para atrasos no desenvolvimento dessa habilidade. Com foco nesses aspectos de estimulação de habilidades, vale destacar que o Ministério de Educação disponibilizou em 2012 um Manual de orientação pedagógica chamado "Brinquedos e brincadeiras de creches" no qual são descritas atividades para estimular diversas áreas do desenvolvimento, entre elas a linguagem (Brasil, 2012). No entanto, medidas preventivas de estimulação relativas a contextos educacionais acabam sendo parciais se outros fatores do contexto familiar e socioeconômico não são levados em consideração, a saber, renda familiar, nível de escolaridade dos pais, acompanhamento de indicadores de saúde da criança, saúde mental dos cuidadores, dentre outros.

Outra área importante no desenvolvimento infantil são as habilidades de coordenação motora. Na presente amostra, foi identificado que o item com maior percentual de falhas entre as 30 crianças com suspeita de atraso motor foi o de montar uma torre e seis cubos 
$(63,30 \%)$ - item que compõe a área de habilidade de coordenação motora fina. Trata-se de um item que demanda da criança habilidades de planejamento, organização viso-espacial, além da própria coordenação motora. Os indicadores de dificuldades na manipulação das torres e execução da tarefa indicam que essas crianças apresentaram dificuldades na organização espacial dos elementos (cubos para formar a torre), mostrando que a princípio podem existir indicadores de suspeitas de atrasos no funcionamento visoperceptivo.

Apesar de ter atingido seu objetivo ao mapear os déficits de habilidades neuropsicomotoras em pré-escolares, o presente trabalho apresenta algumas limitações que devem ser contempladas em estudos posteriores. Sabe-se que o desenvolvimento infantil é um processo complexo e dinâmico, o que sugere a necessidade de múltiplos métodos de avaliação. Neste sentido, embora o Denver II inclua itens de observação direta do comportamento da criança e também relato dos cuidadores, a utilização de um instrumento adicional é recomendável para mapear outros aspectos relacionados ao neurodesenvolvimento. A ausência de normas brasileiras para o Denver II é outra limitação a se destacar, já que nossos dados foram analisados segundo a normatização americana. Desse modo, reiteram-se os argumentos de Rocha, Dornelas e Magalhães (2013), que apontam a importância de investir em pesquisas voltadas para a validação brasileira dos diversos testes importados utilizados para avaliação do desenvolvimento infantil. O tamanho amostral e os critérios de seleção não probabilísticos do grupo também são uma limitação do estudo, embora as taxas de atrasos encontradas tenham sido semelhantes às reportadas em outros estudos nacionais com desenhos metodológicos semelhantes ao nosso.

Por fim, destaca-se que nesta pesquisa, as assistentes maternais das creches foram as informantes. De um lado, foi controlado que essas assistentes exercessem o cuidado pelo menos três meses, antes da avaliação e no mínimo 6 horas diárias, para serem consideradas informantes adequadas. Entretanto, sugere-se para estudos futuros a inclusão da mãe como informante conjuntamente com a assistente maternal. Assim, fatores associados à percepção desses informantes e ao contexto em que a criança se desenvolve poderão ser aspectos incluídos na avaliação. 


\section{Conclusão}

Nesse estudo verificou-se um percentual elevado de crianças com suspeita de atrasos no desenvolvimento neuropsicomotor $(30,00 \%$ de 100 crianças avaliadas), com idades entre 17 e 24 meses. O domínio Motor Grosso do teste foi o menos afetado na amostra. Já o domínio de habilidades motoras finas adaptativas mostrou comprometimentos, em especial nas tarefas relativas à montagem de torres com quatro e seis cubos, sendo um prejuízo que foi identificado tanto no grupo normal como no grupo com suspeita de atraso no desenvolvimento. O domínio com os maiores déficits na amostra total foi $o$ da linguagem. Percentuais elevados de comprometimentos foram verificados em tarefas que avaliaram clareza da fala, número de palavras, habilidades para combinar palavras e nomeação de figuras e ações. Não foram identificadas associações entre a variável relativa ao desenvolvimento (com e sem atraso) e a renda familiar. Embora tenham sido critérios de seleção amostral não probabilísticos os resultados apontaram, para considerar fatores ambientais e socioeconômicos familiares, bem como de fatores relacionados ao contexto das próprias creches como aspectos relevantes para entender uma suspeita de atraso no desenvolvimento. Especial atenção deve ser dada ao domínio da linguagem já que geralmente tende a ser um dos mais comprometidos em crianças assintomáticas e sem queixas de classes socioeconômicas desfavorecidas. O estudo permite concluir a importância de diversos fatores no desenvolvimento infantil, por exemplo, os parâmetros nacionais curriculares da educação infantil, o tipo concreto de prática educacional, o potencial de aprendizagem do aluno, a escolaridade dos pais, renda familiar, a qualidade da estimulação em contextos educacionais e familiares, dentre outros. $\mathrm{E}$ quando estudos de rastreamento mapeiam indicadores de desenvolvimento em ambientes de cuidado primário, como as creches públicas, esses fatores devem ser criteriosamente contemplados.

\section{Referências}

Alckmin-Carvalho, F., Teixeira, M. C. T. V., Brunoni, D., Strauss, V. G., \& Paula, C. S. (2014). Identificação de Sinais Precoces de Autismo Segundo um Protocolo de Observação Estruturada: um Estudo de Seguimento. Psico, 45(4), 502-512.

Aly, Z., Taj, F., \& Ibrahim, S. (2010). Missed opportunities in surveillance and screening systems to detect developmental delay: A developing country perspective. Brain and Development, 32(2), 90-97. DOI: 10.1016/j.braindev.2009.06.004. 
Bates, E., \& Dick, F., (2002). Language, gesture, and the developing brain. Developmental Psychobiology, 40(1), 293-310. DOI: 10.1002/dev.10034.

Barros, R. P., Carvalho, M., Franco, S., de Mendonça, R. S. P., \& Rosalém, A. (2011). Uma avaliação do impacto da qualidade da creche no desenvolvimento infantil. Pesquisa e Planejamento Econômico, 41(2), 214-32.

Belini, A. E. G., \& Fernandes, F. D. M. (2007). Olhar de bebês em desenvolvimento típico: correlações longitudinais encontradas. Revista da Sociedade Brasileira de Fonoaudiologia, 12(3), 165$73 . \quad$ DOI: 80342007000300003

Biscegli, T. S., Polis, L. B., Santos, L. D., \& Vicentin, M. (2007). Avaliação do estado nutricional e do desenvolvimento neuropsicomotor em crianças freqüentadoras de creche. Revista Paulista de Pediatria, 25(4), 337-42. DOI: http://dx.doi.org/10.1590/S0103-05822007000400007

Brasil. (2010). Diretrizes curriculares nacionais para a educação infantil. Ministério da Educação. Brasília, DF.

Brasil. (2012). Brinquedos e brincadeiras de creches: manual de orientação pedagógica. Ministério da Educação. Brasília, DF.

Carvalho, F. A., Paula, C. S., Teixeira, M. C. T. V., Zaqueu, L. D. C. C., \& D'Antino, M. E. F. (2013). Rastreamento de sinais precoces de transtorno do espectro do autismo em crianças de creches de um município de São Paulo. Revista Psicologia: Teoria e Prática, $15(2), 144-54$.

Chang, Z., Lichtenstein, P., Asherson, P. J., \& Larsson, H. (2013). Developmental twin study of attention problems: high heritabilities throughout development. JAMA Psychiatry, 70(3), 311-318. DOI: 10.1001/jamapsychiatry.2013.287

Chiu, S. H., \& DiMarco, M. A. (2010). A pilot study comparing two developmental screening tools for use with homeless children. Journal of Pediatric Health Care, 24(2), 73-80. DOI: 10.1016/j. pedhc. 2009.01.003

Costa, E. F., Cavalcante, L. I. C., \& Dell'Aglio, D. D. (2015). Perfil do desenvolvimento da linguagem de crianças no município de Belém, segundo o Teste de Triagem de Denver II. Revista CEFAC, 17(4), 1090-1102. DOI: 10.1590/19820216201517418514

Couto, M. C. V., Duarte, C. S., \& Delgado, P. G. G. (2008). A saúde mental infantil na Saúde Pública brasileira: situação atual e desafios. Revista Brasileira de Psiquiatria, 30(4), 390-398. DOI: 10.1590/S1516-44462008000400015

Cunha, H. L. (2008). Avaliação do desenvolvimento neuropsicomotor em crianças na rede básica de saúde utilizando o teste de Denver II: identificação de fatores de risco materno. (Tese de 
Doutorado não publicada). Universidade Federal do Rio Grande do Norte, Natal, Rio Grande do Norte, Brasil.

Custódio, Z. A., Crepaldi, M. A., \& Cruz, R. M. (2012). Desenvolvimento de crianças nascidas pré-termo avaliado pelo teste de Denver-II: revisão da produção científica brasileira. Psicologia: Reflexão e Crítica, 25(2), 400-406. DOI: http://dx.doi.org/10.1590/S0102-79722012000200022

Dawson, G. (2013). Early intensive behavioral intervention appears beneficial for young children with autism spectrum disorders. Journal of Pediatrics, 162(5), 1080-81. DOI: 10.1016/j.jpeds.2013.02.049

Figueiras, A. C. M., Pedromônico, M., Sales, L., \& Figueiras, S. (2000). Manual para vigilância do desenvolvimento da criança de 0 a 2 anos de idade na atenção primária à saúde. Secretaria Municipal de Saúde de Belém, Belém, PA.

Figueirêdo, I. G. (2010). Avaliação da qualidade de creches: estudo em um município de São Paulo. (Dissertação de Mestrado não publicada). Universidade Presbiteriana Mackenzie, São Paulo, Brasil.

Fisberg, M., Pedromônico, M. R., Braga, J. A. P., Ferreira, A. M. A., Pini, C., Campos, S. C. C., Campos, S. C. C., Lemes, S. O., Silva, S., Silva, T. M. \& Andrade, T. M. (1997). Comparação do desempenho de pré-escolares, mediante teste de desenvolvimento de Denver, antes e após intervenção nutricional. Revista da Associação Médica Brasileira, 43(2), 99104. DOI: $10.1590 /$ S0104-42301997000200004.

Fonseca, V. D. (2014). Papel das funções cognitivas, conativas e executivas na aprendizagem: uma abordagem neuropsicopedagógica. Revista Psicopedagogia, 31(96), 236253.

Frankenburg, W. K., Dodds, J., Archer, P., Bresnick, B., Maschka, P., Edelman, N., \& Shapiro, H. (1990). Denver II Screening Manual. Denver, CO: Denver Developmental Materials.

Gallahue, D. L., Ozmun, J. C., \& Goodway, J. D. (2013). Compreendendo o Desenvolvimento Motor: Bebês, Crianças, Adolescentes e Adultos. Porto Alegre, RS: Artmed.

Guimarães, A. F., Carvalho, D. V. D., Machado, N. Á. A., Baptista, R. A. N., \& Lemos, S. M. A. (2013). Risk of developmental delay of children aged between two and 24 months and its association with the quality of family stimulus. Revista Paulista de Pediatria, 31(4), 452-458. DOI: 10.1590/S0103-05822013000400006

Halpern, R., Giugliani, E. R. J., Victora, C. G., Barros, F. C., \& Horta, B. L. (2000). Fatores de risco para suspeita de atraso no desenvolvimento neuropsicomotor aos 12 meses de vida. Jornal de Pediatria, 76(6), 421-428. DOI: 10.4067/S037041062002000500016 
Hawa, V. V., \& Spanoudis, G. (2014). Toddlers with delayed expressive language: An overview of the characteristics, risk factors and language outcomes. Research in Developmental Disabilities, 35(2), 400-407. DOI: 10.1016/j.ridd.2013.10.027

Magalhães, L. D. C., Fonseca, K. L., Martins, L. D. T. B., \& Dornelas, L. D. F. (2011). Desempenho de crianças pré-termo com muito baixo peso e extremo baixo peso segundo o teste Denver-II. Revista Brasileira de Saúde Materno Infantil, 11(4), 445-53. DOI: $10.1590 /$ S1519-38292011000400011

Mendes, J. C. D. P., Pandolfi, M. M., Carabetta-Júnior, V., Novo, N. F., \& Colombo-Souza, P. (2012). Fatores associados a alteração da linguagem em crianças pré-escolares. Revista da Sociedade Brasileira de Fonoaudiologia, 17(2), 177-181. DOI: 10.1590/S1516-80342012000200013

Miller, S., Maguire, L. K., \& Macdonald, G. (2011). Home-based child development interventions for preschool children from socially disadvantaged families. Cochrane Database of Systematic Reviews, 12, Art. No. CD008131. DOI: 10.1002/14651858.CD008131.pub2

Peacock, S., Konrad, S., Watson, E., Nickel, D., \& Muhajarine, N. (2013). Effectiveness of home visiting programs on child outcomes: a systematic review. BioMed Central Public Health, 13(17), 1-14. DOI: $10.1186 / 1471-2458-13-17$

Räikkönen, K., Pesonen, A. K., Roseboom, T. J., \& Eriksson, J. G. (2012). Early determinants of mental health. Best Practice \& Research Clinical Endocrinology \& Metabolism, 26(5), 599-611. DOI: $10.1016 /$ j.beem.2012.03.001

Ramos, D. D., \& Salomão, N. M. R. (2013). Desenvolvimento infantil: concepções e práticas de educadoras em creches públicas. Psicologia: Teoria e Prática, 15(3), 200-213.

Rezende, M. A., Beteli, V. C., \& Santos, J. L. F. (2005). Avaliação de habilidades de linguagem e pessoal-sociais pelo Teste de Denver II em instituições de educação infantil. Acta Paulista de Enfermagem, 18(1), 56-63. DOI: 10.1590/S010321002005000100008

Rezende, M. A., Costa, P. S., \& Pontes, P. B. (2005). Triagem de desenvolvimento neuropsicomotor em instituições de educação infantil segundo o teste de Denver II. Escola Anna Nery Revista de Enfermagem, 9(3), 348-55. DOI: 10.1590/S141481452005000300003

Ribeiro, D. G., Perosa, G. B., \& Padovani, F. H. (2014). Fatores de risco para o desenvolvimento de crianças atendidas em Unidades de Saúde da Família, ao final do primeiro ano de vida: aspectos sociodemográficos e de saúde mental materna. Ciência \& Saúde Coletiva, 19(1), 215-226. DOI: 10.1590/141381232014191.1904 
Rocha, S. R., Dornelas, L. de F., \& Magalhães, L. de C. (2013). Instrumentos utilizados para avaliação do desenvolvimento de recém-nascidos pré-termo no Brasil: revisão da literatura. Cardenos de Terapia Ocupacional de São Carlos, 21(1), 109117. DOI: $10.4322 /$ cto. 2013.015

Sabatés, A. L., \& Mendes, L. C. O. (2007). Perfil do crescimento e desenvolvimento de crianças entre 12 e 36 meses de idade que frequentam uma creche municipal da cidade de Guarulhos. Ciência, Cuidado e Saúde, 6(2), 164-170. DOI: 10.4025/cienccuidsaude.v6i2.4143

Sajedi, F., Vameghi, R., \& Kraskian-Mujembari, A. (2014). Prevalence of undetected developmental delays in Iranian children. Child: Care, Health and Development, 40(3), 379-388. DOI: $10.1111 /$ cch. 12042

Scher, M. S. (2013). Normal and abnormal cerebrovascular development: gene-environment interactions during early life with later life consequences. Handbook of Clinical Neurology, 112(1), 1021-1042. DOI: 10.1016/B978-0-444-529107.00021-0

Shan, H., Muhajarine, N., Loptson, K., \& Jeffery, B. (2012). Building social capital as a pathway to success: community development practices of an early childhood intervention program in Canada. Health Promotion International, 29(2), 244-255. DOI: 10.1093/heapro/das063

Shevell, M. I. (2010). Present conceptualization of early childhood neurodevelopmental disabilities. Journal of Child Neurology, 25(1), 120-126. DOI: 10.1177/0883073809336122

Silva, A. C., Engstron, E. M., \& Miranda, C. (2015). Fatores associados ao desenvolvimento neuropsicomotor em crianças de 6-18 meses de vida inseridas em creches públicas do Município de João Pessoa, Paraíba, Brasil. Cadernos de Saúde Pública, 31(9), 1881-1893. DOI: 10.1590/0102-311X00104814

Souza, A. B. G., \& Siqueira, C. N. (2003). Avaliação do desenvolvimento de um grupo de crianças assistidas em creche, usando o teste de triagem de Denver II. Revista Brasileira de Enfermagem, 2(2), 96-103.

Souza, A. L. F., Gouveia, M. T., Almeida, M. D. J. L., \& Torres, C. R. D. (2014). Acompanhamento do crescimento e desenvolvimento infantil na atenção básica. Revista de Enfermagem da UFPI, 2(5), 31-35.

Thal, D., \& Tobias, S. (1994). Relationships between language and gesture in normally developing and late-talking toddlers. Journal of Speech and Hearing Research, 37(1), 157-170. DOI: $10.1044 /$ jshr.3701.157

Valla, L., Wentzel-Larsen, T., Hofoss, D., \& Slinning, K. (2015). Prevalence of suspected developmental delays in early infancy: 
results from a regional population-based longitudinal study. BMC Pediatrics, 15(1), 215-223. DOI: 10.1186/s12887-0150528-Z

Veleda, A. A., Soares, M. C. F., \& Cézar-Vaz, M. R. (2011). Fatores associados ao atraso no desenvolvimento em crianças, Rio Grande, Rio Grande do Sul, Brasil. Revista Gaúcha de Enfermagem, 32(1), 79-85.

Zaqueu, L. C., Teixeira, M. C. T. V., Alckmin-Carvalho, F., \& Paula, C. S. (2015). Associações entre sinais precoces de Autismo, Atenção Compartilhada e Atrasos no Desenvolvimento Infantil. Revista Psicologia: Teoria e Pesquisa, 31(3), 293-302. DOI: $10.1590 / 0102-37722015032243293302$

\section{Endereço para correspondência Maria Cristina Triguero Veloz Teixeira}

Universidade Presbiteriana Mackenzie

Rua da Consolação, 896, prédio 28, $1^{\circ}$ andar, Consolação, CEP 01302-907, São Paulo - SP, Brasil

Endereço eletrônico: mcris@mackenzie.br

Felipe Alckmin-Carvalho

Universidade de São Paulo - USP

Instituto de Psicologia - Departamento de Psicologia Clínica

Av. Professor Mello Moraes, 1721, prédio F, sl 19, Cidade Universitária, CEP 05508030, São Paulo - SP, Brasil

Endereço eletrônico: felipealckminc@gmail.com

\section{Deisy Ribas Emerich}

Universidade de São Paulo - USP

Instituto de Psicologia - Departamento de Psicologia Clínica

Av. Professor Mello Moraes, 1721, prédio F, sl 30, Cidade Universitária, CEP 05508030, São Paulo - SP, Brasil

Endereço eletrônico: deisy.remerich@gmail.com

\section{Priscilla Veloz Cevallos}

Universidade Presbiteriana Mackenzie

Rua da Consolação, 896, prédio 28, 10 andar, Consolação, CEP 01302-907, São Paulo - SP, Brasil

Endereço eletrônico: priscilla.veloz@gmail.com

\section{Cristiane Silvestre de Paula}

Universidade Presbiteriana Mackenzie

Rua da Consolação, 896, prédio 28, $1^{\circ}$ andar, Consolação, CEP 01302-907, São Paulo - SP, Brasil

Endereço eletrônico: csilvestrep09@gmail.com

Recebido em: 08/11/2015

Reformulado em: 27/01/2017

Aceito em: 03/03/2107

\section{Notas}

* Professora Doutora do Programa de Pós-Graduação de Pós-Graduação em Distúrbios do Desenvolvimento do Centro de Ciências Biológicas e da Saúde da 
Universidade Presbiteriana Mackenzie, São Paulo, SP. Tem Graduação em Psicologia pela Universidade de Havana, Cuba. Mestrado em Psicologia e Doutorado em Saúde pela Universidade Federal de Santa Catarina.

** Psicólogo pela Universidade Presbiteriana Mackenzie. Mestre e Doutorando em Psicologia Clínica pelo Instituto de Psicologia da Universidade de São Paulo. Psicólogo clínico no Núcleo Interface de Psicologia (São Paulo). Psicólogo clínico e pesquisador no Programa de Atendimento, Ensino e Pesquisa em Transtornos Alimentares na Infância e Adolescência (PROTAD), serviço do Instituto de Psiquiatria do Hospital das Clínicas da Faculdade de Medicina da Universidade de São Paulo (IPq-HC-FMUSP).

*** Psicóloga. Mestre e Doutoranda em Psicologia Clínica pela Universidade de São Paulo. Professora do Curso de Psicologia do Centro Universitário das Faculdades Metropolitanas Unidas (FMU).

**** Psicóloga pela Universidade Presbiteriana Mackenzie. Bolsista de Iniciação Científica do Cnpq.

***** Professora Doutora do Programa de Pós-Graduação em Distúrbios do Desenvolvimento do Centro de Ciências Biológicas e da Saúde da Universidade Presbiteriana Mackenzie, São Paulo, SP. Tem Graduação em Psicologia pela Universidade Estadual Paulista, UNESP-Assis, São Paulo. Mestrado e Doutorado pelo Departamento de Psiquiatria da Faculdade de Medicina da Universidade Federal de São Paulo/Escola Paulista de Medicina, São Paulo, SP. Pós-Doutorado em Epidemiologia pela Escola Nacional de Saúde Pública da Fundação Oswaldo Cruz (FIOCRUZ), Rio de Janeiro, RJ.

Este artigo de revista Estudos e Pesquisas em Psicologia é licenciado sob uma Licença Creative Commons Atribuição-Não Comercial 3.0 Não Adaptada. 